\title{
COMPARATIVE ADVANTAGE OF KEYHOLE RIGHT FLANK LAPAROTOMY AND VENTRAL MIDLINE CELIOTOMY FOR OVARIOHYSTERECTOMY IN BITCHES
}

\author{
Mohan Acharya ${ }^{1 *}$, Manoj Kumar Sah $^{2}$, Dinesh Kumar Singh ${ }^{3}$, Subir Singh ${ }^{4}$ and Santosh Dhakal ${ }^{1}$ \\ ${ }^{1}$ Institute of Agriculture and Animal Science, Rampur campus, Rampur, Chitwan, Nepal \\ ${ }^{2}$ Veterinary Surgery and Pharmacology, Agriculture and Forestry University, Chitwan, Nepal \\ ${ }^{3}$ Institute of Agriculture and Animal Science, Paklihawa Campus, Paklihawa, Bhairahawa, Nepal \\ ${ }^{4}$ Veterinary Medicine and Public Health, Agriculture and Forestry University, Chitwan, Nepal
}

*Corresponding author email: macharya@uark.edu

\begin{abstract}
The objective was to compare the relative advantage of adopting two ovariohysterectomy techniques: keyhole right flank laparotomy or ventral midline celiotomy, for ovariohysterectomy in bitches. Clinically healthy non pregnant bitches $(n=20 ; 2 \pm 0.5$ year age; $25 \pm 5 \mathrm{~kg}$ weight $)$ were used for the study. Bitches were randomly divided into two groups: group A $(n=10)$ bitches were ovariohysterectomized adopting keyhole right flank laparotomy, whereas in group B $(n=10)$ bitches were ovariohysterectomized adopting ventral midline celiotomy. Time required for completion of surgery, and length of suturing materials required was recorded, blood hematology and fibrinogen levels were analyzed, and post-operative wound healing was monitored. Length of chromic cat gut No. 1-0 (Ethicon; $P<0.01$ ), and vicryl No. 3-0 (Ethicon; $P<0.001$ ) used was more in ventral midline celiotomy compared with keyhole right flank laparotomy; however, similar length of chromic cat gut No. 20 (Ethicon; $P=0.47$ ) was used in both the surgical techniques. Longer time $(P<0.001)$ was required to complete surgical procedure following ventral midline celiotomy compared with keyhole right flank laparotomy. Blood packed cell volume (PCV), total white blood cells (WBC), neutrophils, monocytes, and fibrinogen levels were statistically similar between the two surgical techniques. Wound healing was better in keyhole right flank laparotomy compared with ventral midline celiotomy. Conclusion: Keyhole right flank laparotomy required less time for surgery, less suturing materials and better wound healing compared with ventral midline celiotomy for ovariohysterectomy in bitches.
\end{abstract}

Keywords: bitch; keyhole right flank laparotomy; ovariohysterectomy; ventral midline celiotomy.

\section{Introduction}

Street dogs are creating various problems for people living in major cities of Nepal because these dogs carry rabies, produce noise pollution, fall in traffic accidents and transfer zoonotic diseases (Personal communication, Juliette Cunliffe, founder HART, Nepal). It is estimated that more than sixty zoonotic diseases could be transferred from dogs to humans (Rhindali et al., 2006). Statistical data regarding street dog population in most cities of Nepal is unknown; however, some organizations estimated 22,000 and 1,700 street dogs in Kathmandu and Pokhara respectively (Kakati, 2012; Acharya and Dhakal, 2015). Dogs are highly prolific and their population can be increased in a short period. Therefore it is necessary to control street dogs in order to get rid of zoonotic diseases and other problems.

Non-surgical and surgical sterilization techniques are practiced in dogs to make them unable to reproduce. Hormones and chemicals are used as non-surgical method of sterilization. Progestagens, androgens, and anabolic steroids are commonly used hormones (Max et al., 2014). Chemical sterilization includes the use of cadmium chloride, zinc arginine, glycerol, and lactic acid (Vanderstichel et al., 2014). Castration, ovariectomy, keyhole right flank laparotomy and ventral midline celiotomy are the common surgical sterilization techniques in dogs (Howe, 2006). Several authors have reported drawbacks of using hormones and chemicals (Vanderstichel et al., 2014; Smith, 2014). Surgical sterilization; on the other hand, not only controls population, but also minimizes disease related to reproductive system, mammary tumor and prostatic cancer (Kustriz, 2012).

For surgical sterilization, spaying females instead of castrating males is preferred in most countries including South Asia. It is reasonable because during animal birth control programs an escaped female can mate and produce 15 pups at maximum. If a male escapes, it can mate with several females and produce hundreds of pups. Ovariohysterectomy is a most common surgical 
sterilization in female dogs. This procedure consists of laparotomy with ablation of both ovaries and uterus.

Number of street dogs in Nepal, as well as in most South Asian cities is high. Large number of ovariohysterectomies need to perform in limited time and with limited resources. Keyhole right flank laparotomy and ventral midline celiotomy are the two ovariohysterectomy techniques generally practiced in bitches. This study was done to compare the relative advantages of adopting keyhole right flank laparotomy and ventral midline celiotomy for ovariohysterectomy in bitches.

\section{Materials and Methods}

All animal procedures were done under the supervision of two veterinarians at Veterinary Teaching Hospital, Rampur, Chitwan, Nepal, who takes care of animal welfare and ethical care. Twenty clinically healthy non-pregnant mixed breed bitches ( $\mathrm{n}=20 ; 2 \pm 0.5$ year age; $25 \pm 5 \mathrm{~kg}$ weight), brought for ovariohysterectomy at Veterinary Teaching Hospital, Rampur, Chitwan, were included for the study. Experiment was laid in prospective randomized parallel group clinical trial in which each bitch was placed either in group A: keyhole right flank laparotomy $(n=10)$ or group B: ventral midline celiotomy $(n=10)$. General health conditions was examined and clinical parameters such as heart rate, respiratory rate, and rectal temperature was measured before the surgery. Blood samples were taken before and 5 days after the surgery to determine changes in total white blood cells (WBC), neutrophils, monocytes, blood packed cell volume (PCV), and blood fibrinogen levels. Before the surgery, bitches were pre-medicated with $0.05 \mathrm{mg} / \mathrm{kg}$ atropine sulphate intramuscularly, and were anesthetized with intravenous (IV) administration of xylazine-ketamine combination $(1: 2 \mathrm{v} / \mathrm{v}$; ketamine 50 $\mathrm{mg} / \mathrm{ml}$; xylazine $20 \mathrm{mg} / \mathrm{ml}$ ) via right cephalic vein. Meloxicam, $0.3 \mathrm{mg} / \mathrm{kg}$ weight single dose as a preemptive analgesic and Benzathine penicillin, 40,000 IU/kg body weight single dose as a prophylactic antibiotic were administered intramuscularly before the surgery. Antibiotic powder (Neomycin + Doxycycline) powder was applied topically twice daily after the surgery. Surgical procedures for ventral midline celiotomy and keyhole right flank laparotomy was performed by the methods described below. Time and amount of suture materials required were recorded during the surgery. Time from the start of incision to the end of suturing the external skin layer was considered as time required for completion of surgery. Post-operative wound healing was monitored by external observation 5 days after the surgery.

\section{Procedure for right flank laparotomy}

The bitch was anesthetized by IV administration of xylazineketamine combination $(1: 2 \mathrm{v} / \mathrm{v}$; ketamine $50 \mathrm{mg} / \mathrm{ml}$; xylazine 20 $\mathrm{mg} / \mathrm{ml}$ ) via right cephalic vein and was kept in lateral recumbent position. About $2.5 \mathrm{~cm}$ long dorsoventral incision was made just caudal to the midpoint between the last rib and iliac crest. The incision was extended anteriorly or posteriorly if needed. Skin, subcutaneous fat, external and internal abdominal oblique muscles, transverse abdominal muscle and peritoneum were incised. Double ligation was used to ligate ovarian pedicles and transfixation double ligation was used to encompass entire cervix using chromic cat gut No. 2-0 (Ethicon). Transverse abdominus muscle, internal abdominal oblique muscle and external abdominal oblique muscle were sutured separately following horizontal mattress suture pattern. Peritoneum was incorporated with closure of the transverse abdominus muscle. Chromic cat gut No. 1-0 (Ethicon) was used to suture transverse abdominus muscle and internal and external abdominal muscle. Vicryl 3-0 (Vicryl; Ethicon) was used to close external skin layer following subcuticular suture pattern and terminated with a buried Aberdeen knot. Bitch ovariohysterectomized following keyhole right flank laparotomy is given in Fig. 1.

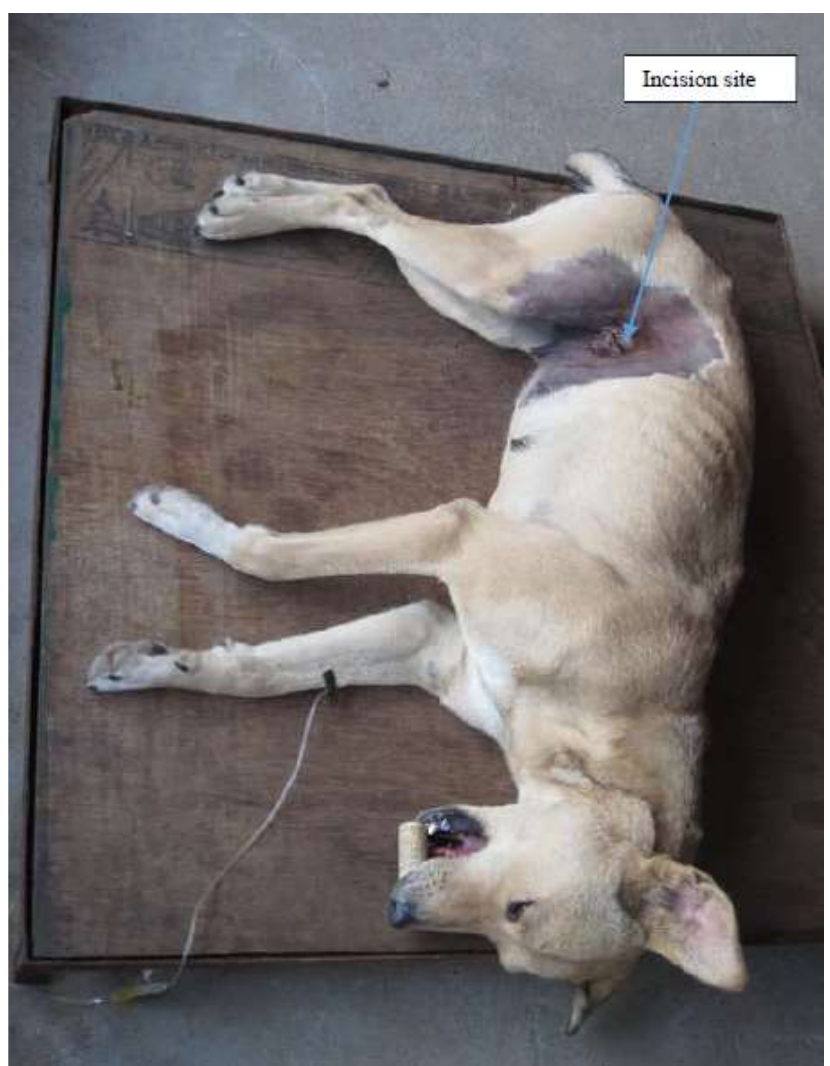

Fig. 1: Bitch ovariohysterectomized following keyhole right flank laparotomy.

\section{Procedure for ventral midline celiotomy}

The bitch was anaesthetized by IV administration of xylazine-ketamine combination $(1: 2 \mathrm{v} / \mathrm{v}$; ketamine 50 $\mathrm{mg} / \mathrm{ml}$; xylazine $20 \mathrm{mg} / \mathrm{ml}$ ) via right cephalic vein and was place in dorsal recumbent position. Incision was made $2 \mathrm{~cm}$ caudal to the umbilicus. Skin, subcutaneous tissue, linea alba and peritoneum was incised. At first about $4 \mathrm{~cm}$ long incision was made but later slightly increased anteriorly or posteriorly in order to expose ovaries as well as junction of cervix and uterine body for easy ligature placement. Ligation for ovarian pedicles and cervix was done in the same way as described for right flank laparotomy. Peritoneum and linea alba was closed using chromic cat gut No. 1-0 (Ethicon). Simple interrupted suture pattern was used to suture linea alba. Buried intradermal suture pattern was used to close skin using vicryl No. 3-0 (Vicryl; Ethicon). Bitch ovariohysterectomized following ventral midline celiotomy is given in Fig. 2. 


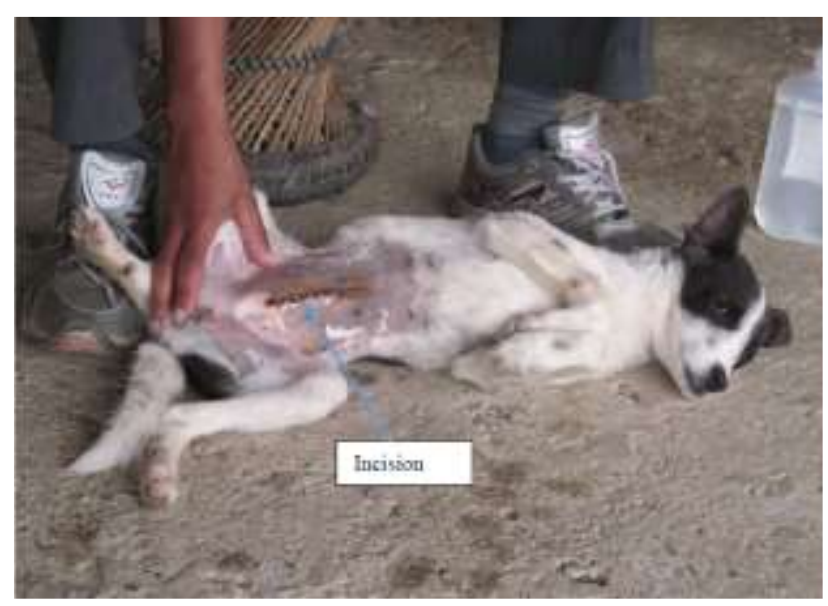

Fig. 2: Bitch ovariohysterectomized following ventral midline celiotomy.

\section{Statistical analysis}

Data were analyzed by t-test (using statistical package R2010). Values are presented as mean and standard deviation.

\section{Results}

White blood cells, neutrophils, monocytes, and fibrinogen levels increased and PCV decreased in both the ovariohysterectomy techniques; however, changes in values were not statistically different $(P>0.05)$ between the surgical techniques (Table 1). Ventral midline celiotomy required more time $(P<0.001$; mean $=49.4 \pm 0.59$ minutes $)$ compared with keyhole right flank laparotomy ( mean $=26.2$ \pm 0.76 minutes). Similarly, chromic cat gut No. 1-0 (Ethicon) was used more $(P<0.01$; mean $=38 \pm 0.69 \mathrm{~cm})$ in ventral midline celiotomy compared with right flank laparotomy $($ mean $=29.7 \pm 0.85 \mathrm{~cm})$. Use of chromic cat gut No. 2-0 (Ethicon) was statistically similar $(P=0.477)$ between ventral midline celiotomy $($ mean $=20 \pm 0.91 \mathrm{~cm})$ and keyhole right flank laparotomy (mean $=19 \pm 0.89 \mathrm{~cm}$ ). Ventral midline celiotomy required more vicryl No. 3-0 (Ethicon; $P<0.001$; mean $=26.1 \pm 0.57 \mathrm{~cm}$ ) compared with keyhole right flank laparotomy $(17.3 \pm 0.76 \mathrm{~cm})$.

\section{Discussion}

In this study we used xylazine and ketamine as a general anesthesia, procaine penicillin as a prophylactic antibiotic, and chromic cat gut (1-0, 2-0) and vicryl (3-0) as a suture materials. The general anesthesia, prophylactic antibiotic, and suture materials used in this study are also commonly used during ovariohysterectomy in street dog animal birth control programs (Reece et al., 2012). In India alone over one hundred thousand street dogs are sterilized every year following this protocol (www.awbi.org).

All bitches used in this study were non-pregnant Mongrel breed of similar age and, therefore, were more or less uniform in size. Mixed solution of xylazine: ketamine (ketamine $50 \mathrm{mg} / \mathrm{ml}$; xylazine $20 \mathrm{mg} / \mathrm{ml} ; 1: 2 \mathrm{v} / \mathrm{v}$ ) was used for both induction and maintenance anesthesia. First induction dose was given on the basis of weight (xylazine: ketamine; $1 / 2 \mathrm{v} / \mathrm{v} ; 2 \mathrm{~mL} \mathrm{I} / \mathrm{V}$ at the rate of $20 \mathrm{Kg}$ weight). First tapering dose was given roughly 15 minutes after induction dose ( 0.5 to $1 \mathrm{~mL} \mathrm{I} / \mathrm{V}$ depending on size) and second tapering dose $(0.5 \mathrm{~mL} \mathrm{I} / \mathrm{V}), 10$ minutes after the first tapering dose. Depending upon the length of surgery third and fourth tapering doses each of $0.5 \mathrm{~mL}$ were given in 10 minutes interval. Since time is correlated with amount of anesthetic we dropped amount of anesthetics used. In this trial, we found a difference in time required for the completion of surgery, between keyhole right flank laparotomy and ventral midline celiotomy for ovariohysterectomy in bitches, less time being required in keyhole right flank laparotomy. Studies done in India concluded similar results; however, time required was much lower compared with our trial (keyhole right flank, $7.16 \pm$ 0.65 minute; and ventral midline $11.50 \pm 0.42$ minute; Sutton and Johnstone, 1997; Murthy et al., 2012). Difference could be due to size of the dogs and experience of the technicians performing surgery.

Table 1: Changes in blood hematology and fibrinogen levels before and 5 days after the surgery. Values expressed in mean $\pm \mathrm{SD}$.

\begin{tabular}{|l|l|l|l|l|}
\hline Parameters & $\mathrm{KRFL}^{2}$ & $\mathrm{VMC}^{3}$ & $P$-value & significance \\
\hline Increase in $\mathrm{WBC}^{4}$ (cu.mm) & $990 \pm 10$ & $1045 \pm 11$ & $P>0.05$ & $\mathrm{NS}^{1}$ \\
\hline Decrease in $\mathrm{PCV}^{5}(\%)$ & $2.40 \pm 0.11$ & $3.30 \pm 0.09$ & $P>0.05$ & $\mathrm{NS}^{1}$ \\
\hline Increase in fibrinogen level (g/dl) & $0.29 \pm 0.01$ & $0.22 \pm 0.01$ & $P>0.05$ & $\mathrm{NS}^{1}$ \\
\hline Increase in Neutrophil (\% of WBC) & $2.80 \pm 0.09$ & $2.10 \pm 0.08$ & $P>0.05$ & $\mathrm{NS}^{1}$ \\
\hline Increase in Monocytes (\% of WBC) & $1.40 \pm 0.07$ & $1.50 \pm 0.06$ & $P>0.05$ & $\mathrm{NS}^{1}$ \\
\hline
\end{tabular}

midline celiotomy, ${ }^{4} \mathrm{WBC}=$ White blood cells, ${ }^{5} \mathrm{PCV}=$ packed cell volume. 
The muscle layer encountered during the two ovariohysterectomy techniques are different and vary in numbers i.e., more muscle layers encountered in keyhole different and vary in numbers i.e., more muscles layers encountered in keyhole right flank laparotomy compared with ventral midline celiotomy. Therefore, length of suturing material and time of spaying were considered as major cost factors for ovariohysterectomy. In this study we evaluated length of suture materials required in each ovariohysterectomy technique. Studies regarding the detailed evaluation of suture materials required in each surgical technique for ovariohysterectomy in bitches are rear or are not done. We found less chromic cat gut No. 1-0 (Ethicon) and vicryl 3-0 (Ethicon) required in keyhole right flank incision compared with ventral midline celiotomy whereas equal amount cat gut No. 2-0 (Ethicon) were used in both techniques. It is reasonable that similar amount of cat gut No. 2-0 (Ethicon) was used in both the ovariohysterectomy techniques as this suture material was used only during ligation of ovarian pedicle and to encompass cervix. Reason for use of more chromic cat gut No. 1-0 in ventral midline celiotomy compared with keyhole right flank could be due to larger incision length in midline celiotomy. In both the techniques, vicryl 3-0 was used only during closure of outermost skin and is reasonable that ventral midline celiotomy required more compared with keyhole right flank laparotomy. Keyhole right flank required less time and suture materials compared with ventral midline celiotomy and hence this could be the reason that several authors concluded keyhole right flank laparotomy as a cheaper ovariohysterectomy technique for bitches (Murthy et al., 2012; Reece et al., 2012).

Total WBC, monocytes, neutrophils, and blood fibrinogen levels increased after the surgery, however, these changes were similar between the techniques. Increase in fibrinogen level could be due to various inflammatory phases of wound healing (Sutton and Johnstone, 1997). Increase in neutrophils after the surgery could be due to neutrophilia that is essential after the surgery in order to clean dead tissue inside the body and monocyte increase could be attributed to its cytokine regulation during antigen processing in the cell (Greenhalgh, 1998; Aiello, 2000; Khan et al., 2005). In both the techniques, there was slight bleeding during the surgery. This could have decreased total RBC and loss could not have recovered during the measurement taken on fifth day of surgery. Therefore, decrease in PCV after surgery could be due to blood loss during surgery. Though there was increase in total WBC, neutrophils, monocytes and fibrinogen levels, changes were not statistically different between the techniques. This implies that healing occurred in both the techniques, and the rate of healing on the basis of measured blood parameters was similar in both ovariohysterectomy techniques.
Post-operative monitoring showed that most of the bitches on keyhole right flank laparotomy group were active and normal; there was no redness, soiling and discharges in and around the incision area. Some bitches in ventral midline celiotomy group had redness around incised area and had little sero-saunguinous discharges. This shows that chances of post-operative complications could be more in ventral midline celiotomy compared with keyhole right flank laparotomy. This could be due to longer incision length in ventral midline celiotomy compared with keyhole right flank laparotomy (Reece et al., 2012). Additionally incision area in ventral midline is linea alba which lacks vascularity and this place is in constant pressure from stomach. No vascularity, and constant pressure could have slowed down healing process in ventral midline compared with keyhole right flank laparotomy.

In conclusion, Street dog population is increasing day by day in most cities of Nepal, as well as in South Asia. Street dogs are the source of zoonotic diseases including rabies. It is an urgent need to control dog population in a given limited time and limited resources. Ovariohysterectomy in bitches controls street dog population in scientific and humane manner. Keyhole right flank laparotomy and ventral midline celiotomy are the two ovariohysterectomy techniques generally practiced in dogs. On the basis of our observations we found that keyhole right flank laparotomy required less time, less suture materials, quicker wound healing, and has less post-operative complication compared with ventral midline celiotomy. However, for keyhole right flank ovariohysterectomy, surgeon need to be skilled. Disadvantages of adopting keyhole right flank ovariohysterectomy includes; visualization of surgical parts during the surgery is difficult and management of postoperative complications is hard.

\section{Conflict of interest}

The authors declares that there is not conflict of interest.

\section{Reference}

Acharya M and Dhakal S (2015) Survey on Street Dog Population in Pokhara Valley of Nepal. Bangl. J. Vet. Med. 13: 65-70. DOI: $10.3329 /$ bjvm.v13i1.23722

Aiello SE (2000) The Merck Veterinary Manual. $10^{\text {th }}$ edition. Merck and Co, USA, pp 1211.

Animal Welfare Board of India (2009). Standard operating procedures for sterilization of stray dogs under the animal birth control programme. Available online http://www.awbi.org/awbi pdf/SOP.pdf. Accessed November, 2015.

Greenhalgh DG (1998) The role of apoptosis in wound healing. Int J Biochem Cell Biol. 30: 1019-1030. DOI: 10.1016/S 1357-2725(98)00058-2

Howe LM (2006) Surgical methods of contraception and sterilization. Theriogenology. 66: 500-509. DOI: 
$10.1016 / \mathrm{S}$

$1357-2725(98) 00058-2$

10.1016/j.theriogenology.2006.04.00

Kakati K (2012) Street dog population survey, Kathmandu 2012. Available

online https://animalnepal.files.wordpress.com/2013/09/dogsurvey-kathmandu-valley-2012.pdf

Khan AI Heit B Andonegui G Colarusso P and Kubes P (2005) Lipopolysaccharide: A p38 MAPK-dependent disrupter of neutrophil chemotaxis. Microcirculation. 12: 421-432. DOI: $10.1080 / 10739680590960368$

Kustriz MVR (2012) Effects of surgical sterilization on canine and feline health and on society. Reprod in Domest Anim. 47: 214-222. DOI: 10.1111/j.1439-0531.2012.02078.x

Max A Piotr J Dobrzynski A and Rijsselaere (2014) Non-surgical contraception in female dogs and cats. Acta Sci. Pol. Zootechnica. 13: 3-18.

Murthy VC Murthy CAN Jamuna KV and Nagaraja (2012) Comparision of different laparotomy technique of ovariohysterectomy and post-surgical complications in dogs. Indian Journal of Canine Practice. 4: 116-118.
Reece JF Nimesh MK Wyllie RE Jones AK and Dennison AW (2012) Description and evaluation of a right flank, minilaparotomy approach to canine ovariohysterectomy. The Vet Rec. 171: 248-248. DOI: 10.1136/vr.100907

Rhindali L Biggeri A Carbone S Musella V Catelan D Veneziano $\mathrm{V}$ and Cringoli G (2006) Canine fecal contamination and parasitic risk in the city of Naples (Southern Italy). BMC Vet Res. 2: 29-35. DOI: 10.1186/1746-6148-2-29

Smith AN (2014) The Role of neutering in cancer development. Vet Clin $N$ Am-Small. 44: 965-975. DOI: 10.1016/j.cvsm.2014.06.003

Sutton RH Johnstone M (1997) The value of plasma fibrinogen estimation in Dogs. A comparison with total leucocytes and neutrophil count. JSAP. 10: 277-281. DOI: 10.1080/00480169.1975.34186

Vanderstichel R Forzan MJ Perez GE Serpell JA and Garde E (2014) Changes in blood testosterone concentration after surgical and chemical sterilization of male free-roaming dogs in southern Chile. Theriogenology. 83: 1021-1027. DOI: $10.1016 /$ j.theriogenology.2014.12.001 